\title{
Landscape structure and boundary effects determine the fate of mutations occurring during range expansions
}

\author{
OJ Burton and JMJ Travis \\ Institute of Biological and Environmental Sciences, University of Aberdeen, Aberdeen, UK
}

\begin{abstract}
The interplay between the spatial dynamics of range expansion and evolutionary processes is receiving considerable attention. Recent theory has demonstrated that mutations occurring towards the front of a spatially expanding population can sometimes 'surf' to high frequency and spatial extent. Here, we extend this work to consider how the fate of a novel mutation is influenced by where and when it occurs. Specifically, we are interested in establishing how the origin of a mutation relative to a habitat edge influences its dynamics, and in understanding how this is mediated by the behaviour of individuals at those boundaries. Using a coupled-map lattice model, we demonstrate that the survival probability, abundance and spatial extent of surviving mutants can depend on their origin. An edge effect is often
\end{abstract}

observed and can be quite different both qualitatively and quantitatively depending on the behavioural rules assumed. Mutations, especially those that are deleterious, that arise at a habitat edge with reflective boundary conditions can be many more times likely to survive for substantial periods of time than those that arise away from the edge. Conversely, with absorbing boundary conditions, their survival is greater when they arise well away from the edge. Our results clearly illustrate that landscape structure, habitat edges and boundary conditions have a considerable influence on the likely fate of mutations that occur during a period of range expansion.

Heredity (2008) 101, 329-340; doi:10.1038/hdy.2008.56; published online 2 July 2008

Keywords: evolution; invasion; range shifting; evolvability; climate change; mutations

\section{Introduction}

Landscape genetics is an emerging field of research that seeks to understand how the structure of habitat and matrix influences the genetics of spatially structured populations (Manel et al., 2003; Storfer et al., 2007). The majority of work in this area has focussed on describing and understanding spatial patterns of neutral genetic variability and has considered populations with stable biogeographic ranges. However, it is increasingly recognized that this may provide a rather poor indication of how adaptive variation is structured across a landscape. In addition, many species are already shifting their biogeographic ranges as the climate changes (Parmesan and Yohe, 2003), and others are undergoing range expansions as they invade regions where they are nonnative. Thus, there is considerable current interest in the biology of dynamic species' ranges, including genetic (for example, Lee, 2002; Edmonds et al., 2004; Klopfstein et al., 2006) and evolutionary processes (for example, Hänfling and Kollmann, 2002; Travis and Dytham, 2002; Lambrinos, 2004; Simmons and Thomas, 2004). Theoretical models, within landscape ecology, have demonstrated that the structure of a landscape, in terms of the

Correspondence: OJ Burton, University of Aberdeen, Zoology Building, Tillydrone Avenue, Aberdeen AB24 2TZ, UK.

E-mail: olivia.burton@abdn.ac.uk

Received 14 August 2007; revised 1 May 2008; accepted 14 May 2008; published online 2 July 2008 extent and pattern of habitat availability, can influence the spatial population dynamics of both invasive spread (With, 2002) and range shifting in response to climate change (McInerny et al., 2007). These methods can readily be extended to incorporate population genetics to determine how different key features of a landscape, such as habitat boundaries, influence evolutionary processes.

Ecological boundaries arise across a wide range of habitats and landscapes and have a diverse impact on species' and community dynamics (Cadenasso et al., 2003 and references within). The response of individuals to habitat edges is species specific. Species in many groups disperse their seeds, or offspring, passively and have little, or no, control over where they move to. For example, a large proportion of plant seeds are dispersed by wind (for example, Tackenberg et al., 2003), whereas many species of spiders, spider mites and moth larvae utilize a high-risk dispersal strategy using aerial balloons (for example, Bonte et al., 2003; Bell et al., 2005). These passively dispersed species will show little response to habitat boundaries, whereas those species that exert more control over their dispersal, for example, most birds, mammals and amphibia and many insects, will exhibit very different movement behaviours close to boundaries than they typically do away from them (for example, Stevens et al., 2006; Chapman et al., 2007). Spatial models are a useful tool for determining how behaviour at these boundaries might influence population or community dynamics. By assuming different 
rules at these edges (Fagan et al., 1999), models can incorporate the different behaviours that organisms may exhibit at habitat boundaries. Three different boundary conditions are widely implemented in simulation and mathematical modelling-absorbing, reflective and periodic (sometimes also termed cyclic or torus). Absorbing boundary conditions lead to individuals being completely removed from the population when they disperse to a region or patch beyond the boundary and are most appropriate for modelling passively dispersed organisms. Reflective conditions result in impermeable edges, so individuals will 'bounce off' the boundary and will be positioned in a patch before that edge. Organisms that disperse actively, and react to habitat edges by changing their movement behaviour, are more appropriately modelled using reflective conditions. Periodic boundary conditions differ in that they are a theoretic construct designed to approximate a system with an infinite number of patches so, for example, individuals that disperse off the left and top of a lattice will reappear at the right and bottom. Many theoretical studies present results that are all obtained using a single boundary condition, in some cases with a statement suggesting that results are similar when alternative conditions are employed. For example, studies have found boundary conditions to have no (Molofsky et al., 2002) or little effect (Comins et al., 1992; Kean and Barlow, 2000), whereas others quote any effect to have led to similar results (Etienne et al., 2002; Leblois et al., 2006). Few studies present rigorous analyses of the potential for different boundary conditions to generate different results. Here we conduct simulations to assess how habitat edges, together with boundary conditions, affect the dynamics of mutations that occur during a range expansion.

There has been considerable recent interest in understanding how the spatial dynamics of range expansions determine patterns of neutral genetic variability in recently colonized regions (for example, Ibrahim et al., 1996; Austerlitz and Garnier-Géré, 2003; Bialozyt et al., 2006). Complementing this, Edmonds et al. (2004) and Klopfstein et al. (2006) have investigated in some detail the fate of single novel mutants that occur during range expansion (Edmonds et al., 2004; Klopfstein et al., 2006). Neutral mutations that arise towards the front of a range expansion can sometimes 'surf' on the wave of advance, attaining much higher frequency and spatial extent than would be anticipated for a stationary population (Edmonds et al., 2004; Klopfstein et al., 2006). Occurring close to the expanding front confers two key advantages on a novel mutation: firstly, it is likely to suffer from reduced density dependence, as demes towards the front are likely to be below carrying capacity; secondly, being close to the front makes it more likely that its progeny will become the first individuals to colonize patches beyond the existing front, themselves then benefiting from little intraspecific competition. Klopfstein et al. (2006) suggested that this surfing effect should increase the adaptive potential of range-expanding populations. However, they only considered the fate of neutral mutations and, given the sometimes poor correlation between patterns of neutral and adaptive variability (for example, Knopp et al., 2007), there is a need for further studies that consider the behaviour of non-neutral mutations. A recent, direct extension of the Edmonds et al. (2004) approach has demonstrated that the surfing effect is important regardless of a mutant's fitness effect (Travis et al., 2007); mutations occurring close to the front of an expanding range always have a higher probability of survival, on average reach a higher total abundance and are present within a greater number of demes. Travis et al. (2007) also showed that the spatial distribution of a surviving mutant differs considerably depending on its fitness effect, with most surviving beneficial mutations having their centroid close to where they originated, whereas most surviving deleterious mutations have a spatial distribution concentrated close to the expanding front. In a further recent extension, Burton and Travis (2008) developed a two-locus model, incorporating sign epistasis, and demonstrated that fitness peak shifts are more likely during range expansion due to the importance of this surfing effect for the survival and abundance of deleterious mutations. Here, we extend the methods used by Travis et al. (2007) to consider how surfing dynamics are influenced by landscape structure. In particular we are interested in how habitat edges, and the behaviour of individuals at those edges, determine the dynamics of a novel mutation.

We use a coupled-map lattice to establish how elements of landscape structure and individual behaviour in response to habitat edges affect survival and subsequent population dynamics of novel mutations occurring within an expanding range.

We study the effect of the initial location of a mutant, relative to the edge of the lattice, for three different boundary conditions. We determine how the probability of mutant survival to different time points is modified by its initial location and also assess how the frequency and spatial extent of surviving mutants may vary according to where and when they first occurred. We then run two sets of simulations to assess how landscape structure might modify the dynamics of mutations occurring during range expansions. In the first set of simulations, a single block of habitat is made unsuitable within the centre of the lattice, whereas in the second a short, narrow corridor provides the only migration route between two large areas of intact habitat. We discuss the implications of our results for populations expanding their ranges across complex landscapes and highlight some directions that future studies might pursue.

\section{The model}

We use a spatial model to simulate the occurrence of mutations within an expanding range under different boundary conditions. This model is an extension of recent studies investigating the dynamics of neutral (Edmonds et al., 2004; Klopfstein et al., 2006) and nonneutral (Travis et al., 2007; Burton and Travis, 2008) mutations arising at expanding range margins. Here, we consider the effects of employing different boundary conditions and the position of a mutation's origin on the fate and distribution of both neutral mutations and those that alter the relative fitness of individuals. We also investigate the potential role of landscape structure, running two sets of simulations on arenas modified to incorporate some habitat complexity.

\section{Spatial dynamics}

We simulate the range expansion of haploid individuals on a rectangular two-dimensional stepping stone grid 
(20 rows $\times 800$ columns). A population comprising a single genotype (subsequently referred to as the ancestral genotype) is introduced into the left-most 50 columns, and for 20 generations it is restricted to this region. After these 20 generations, the population is allowed to expand its range. A novel mutant is introduced at a pre-specified location $\Delta T$ generations after that deme has been colonized by the ancestral genotype. Unless stated otherwise, novel mutants are introduced at $x=100$. In most of the results presented, the mutant is introduced as soon as the patch is colonized, that is, $\Delta T=0$. Each simulation runs for 100 generations after the novel mutant is introduced or until the mutant becomes extinct. We investigate the fate of single mutations that occur at the edge of the range expansion and collect data to ascertain how both the survival probability and spatial dynamics of a novel mutant depend on where it arises. Figure 1 schematically illustrates the key features of the model.

The abundance of individuals within each deme is limited by a carrying capacity $K$. In each generation, individuals produce a number of offspring drawn at random from the Poisson distribution with mean $R$. Offspring inherit the parental genotype and always survive to the next generation if the number of individuals within each patch is not greater than $K$. If $K$ is exceeded, then the number of mutant and nonmutant juveniles that survive to the next generation is drawn at random from Wallenius' non-central hypergeometric distribution (Wallenius, 1963). Using this distribution provides a computationally efficient way of simulating biased lottery competition. Four parameters are required to make each random draw from this distribution: the number of adults that will be present in the next generation $(K)$, the number of mutants in the juvenile pool, the total number of juveniles and the bias. If the bias is set to 1.0, the model simulates the dynamics of a neutral mutation. Values of this parameter greater
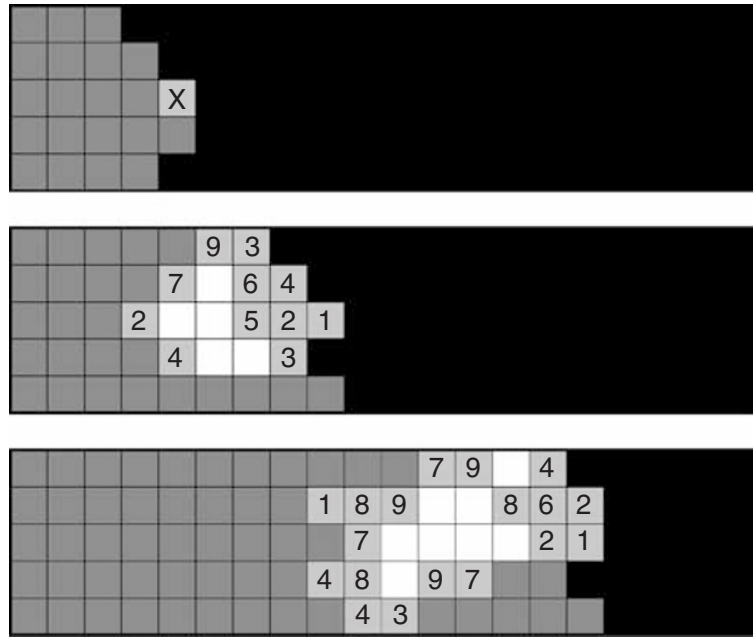

Figure 1 A simplified schematic illustrating the essential dynamics incorporated in the model. A population of the ancestral genotype is allowed to expand from the left-hand side of the arena into black, unoccupied space. A single mutant is introduced into patch $X$ as soon as the population colonizes that location. Here, the ancestral genotype is depicted in dark grey, the number of mutant individuals in patches containing both genotypes is indicated in light grey cells, and white illustrates patches containing only mutant individuals. than 1.0 are used to simulate the dynamics of beneficial mutations, whereas values less than 1.0 are used for deleterious mutations. For example, an individual with a bias of 1.1 is $10 \%$ more likely to survive to the next generation than an individual with a bias of 1.0. Surviving juveniles disperse with probability $d$, and emigrating individuals move at random to one of the nearest four neighbouring patches. In all the results reported here, $K=10$. We have conducted a sensitivity analysis using $K=5,10,30,50$ and 100 . Qualitatively the results are extremely similar, as has been previously reported by Klopfstein et al. (2006).

\section{Behaviour at edges}

We run simulations using three different boundary conditions. We initially run the model with absorbing boundaries where individuals dispersing off the edge of the lattice are removed from the population. A second set of simulations is undertaken using reflective boundaries. Here, individuals that should disperse to a deme off the top or bottom of the lattice are instead relocated to one of the remaining nearest neighbouring cells to the origin, chosen at random, nearest to the original destination in the neighbouring horizontal plane. The final set of simulations is carried out using periodic boundary conditions to simulate an effectively infinite range width. Here, individuals leaving the top of the lattice re-enter at the bottom with an equivalent $x$-coordinate and vice versa. Individuals that disperse off the back of the expanding range (that is, attempt to move to the left from column 0) are removed from the population so that they do not reappear on the right hand side of the lattice.

\section{The simulation experiments}

We run simulations for each of three mutant fitness values for each of the boundary conditions. The ancestral genotypes have a fitness of 1.0 and mutant individuals are, in turn, given fitness values of 0.80 (for deleterious mutations), 1.0 (for neutral mutations) and 1.25 (for beneficial mutations). Here, set values of $K=10$ and $R=1.8$ are used for every simulation. For each combination of fitness value and boundary condition, we run simulations varying the $y$-coordinate from 0 to 9 . Mutants are always introduced at $x=100$. For each introduction coordinate, the model is repeated until we have 100 successful simulations. A successful simulation is defined as a run of the model where at least one individual with the mutant genotype is present within the population for 100 generations following the mutant's introduction. This provides sufficient data to assess how both the survival probability and subsequent dynamics of a mutant varies depending on where it arises. To aid interpretation of the genetic results, we also collect data on the position of the population's range front in relation to the habitat edge. For all three boundary conditions we run the simulations, and after 100 generations of range expansion we determine the $y$-coordinate(s) of maximum range expansion. We count the number of times that the front is present at each $y$-coordinate.

For each simulation, data on the total number of individuals, the number of mutants, the number of demes occupied by mutants and the survival probability 
of mutant individuals are collected for each position from the top $(y=0)$ to the centre $(y=9)$ of the lattice. The spatial distribution of the mutants is also investigated in each of the simulations by recording the average $x$ - and $y$-coordinate of all mutant individuals to give the centroid of each mutant population. The coordinates of the demes occupied by mutant individuals furthest from this centroid are also recorded. Simulations are carried out for dispersal probabilities of $0.1,0.2$ and 0.3. We also run a further set of simulations varying $\Delta T$ and additionally collect equivalent data to assess how the fate of mutations occurring in a stationary range depends on where they occur in relation to the habitat edge.

\section{Landscape structure}

Landscapes are very rarely homogenous at any spatial scale, and heterogeneity in patch suitability may play an important role in determining the fate of mutations according to where they arise. We run simulation experiments to establish how two simple and abstract patterns of habitat availability influence the dynamics of the mutants during range expansion. These simulations are very similar to those already described, except that now a set of patches are deemed unsuitable for the species (shown in red in Figure 2). We also vary both the $x$ - and $y$-coordinates of mutant introduction in these simulations. Range expansion is initiated as in previous simulations on a 20 rows $\times 800$ columns grid and data are collected on the survival and subsequent dynamics of mutants arising in all patches where $100 \leqslant x \leqslant 112$ and $0 \leqslant y \leqslant 19$. Information is collected for these $x$-coordinates, as they are those closest to the habitat feature where the effects are likely to be greatest. Mutations introduced outside of these values would be affected less by the landscape heterogeneity. Pragmatically, we are limited in how broad the area of the landscape is over which to collect data, as it is a computer-intensive process. For example, to draw a single pixel on Figure 7, it required 62500 simulations (before 100 mutants had survived for 100 time steps), and in total we used 20 processors over almost 3 months to obtain all the data presented in this article.

\section{Results}

\section{Survival and dynamics of mutants}

Beneficial mutations always have a higher probability of survival than neutral mutations, which in turn survive more frequently than deleterious mutations. Mutants, regardless of fitness effect, arising close to the front sometimes surf to high frequency and spatial extent (Travis et al., 2007; Burton and Travis, 2008). The survival probability of deleterious mutations occurring on the front of an expanding range can be relatively high, up to nearly $20 \%$ (Figure 3a), and is conditional on this surfing behaviour. This leads to surviving deleterious mutants (Figure 3c) on average travelling much further than either neutral (Figure 3f) or beneficial mutants (Figure 3i), which both have very similar maximum $x$-displacements. So great is the effect of this surfing behaviour that surviving deleterious mutants on average have a greater patch occupancy and total population size than surviving neutral mutants (Table 1, Figure 3). Surviving beneficial mutants always have a higher patch occupancy and total population size than surviving neutral or deleterious mutants (Table 1 ).

The probability of natal dispersal influences both the probability of mutants surviving and the spatial dynamics of those that survive (Table 1). Survival probability always decreases with increasing dispersal and this effect is greatest for deleterious mutants. For example, there is a reduction in survival probability of deleterious mutants from 12.02 to $7.91 \%$ when dispersal is increased from 0.1 to 0.3 , whereas for beneficial mutants, the equivalent comparison is 63.76 and $59.58 \%$ (Table 1). The maximum $x$-displacement of mutants always increases when dispersal is more frequent, and for all three mutant types, we observe roughly a doubling in distance travelled when dispersal increases from 0.1 to 0.3 (Table 1). Dispersal probability affects both patch occupancy and total population size very similarly, and for the three mutant types, there is always an increase in both as dispersal increases. However, in this case, there is a quantitative difference in the effect of dispersal depending on fitness effect, with a greater increase in both patch occupancy and population size for a
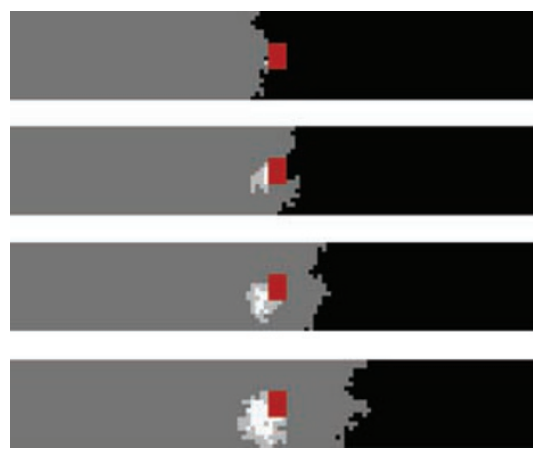

100

b

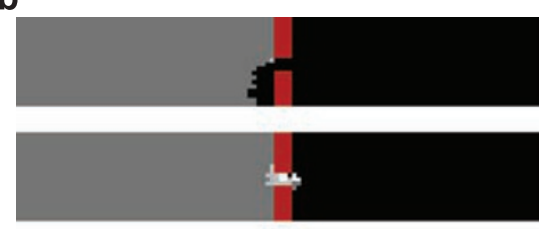

160

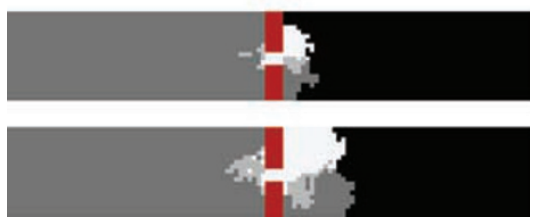

Figure 2 Snapshots from two independent simulations illustrating the typical dynamics observed. (a) Snapshots for simulations conducted with a patch of unoccupied habitat. (b) Snapshots of a simulation incorporating a habitat corridor. Here, red indicates unavailable habitat, black unoccupied space and the darker shade of grey shows patches where only the ancestral genotype is present. White depicts demes where only the mutant is present and mid-grey depicts those demes where both the genotypes occur. A population made up entirely of the ancestral genotype is allowed to expand from the left of the arena and, after 100 time steps, a mutation occurs at the range front. The figures illustrate the spatial spread and extent of the expanding population and the mutant cloud at three further time points. These graphics were generated for beneficial mutants (fitness $=1.25$ ), $K=10$ and $R=1.8$. 

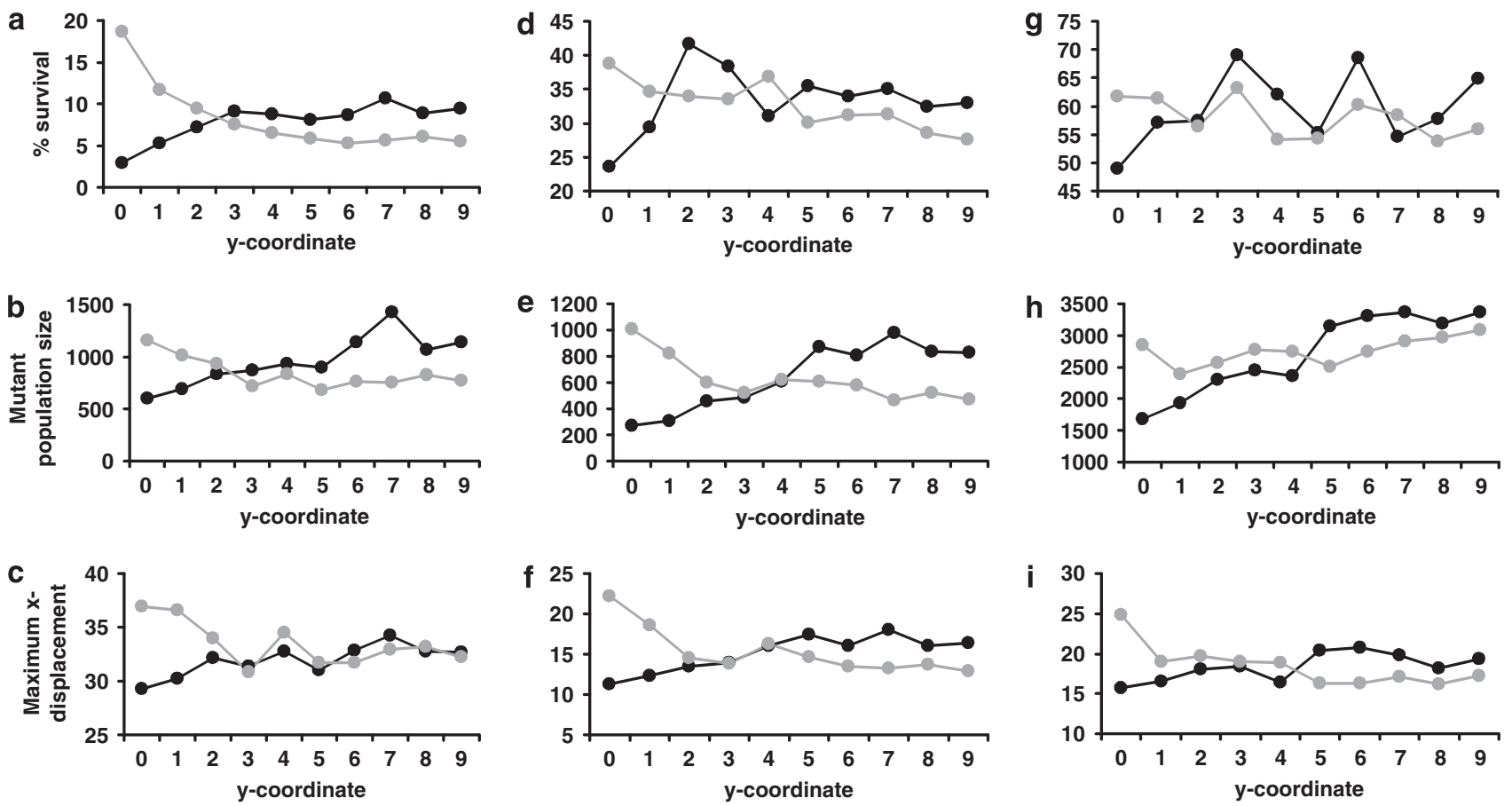

Figure 3 Edge effects influence survival probability, mutant population size and maximum $x$-displacement. The nature of the edge effect is determined by boundary condition and mutant fitness. In each of the plates, grey indicates results obtained for reflective and black for absorbing boundary conditions. Results for $(\mathbf{a}-\mathbf{c})$ deleterious, $(\mathbf{d}-\mathbf{f})$ neutral and (g-i) beneficial mutants. All results were generated for $K=10$ and $R=1.8$. For illustrative purposes, the $y$ axis scale varies between graphs.

beneficial compared with neutral and deleterious mutations (Table 1).

\section{Edge effects and boundary conditions}

Both the location at which a mutant arises relative to habitat edge and the boundary condition can have a major influence on the likely survival and spatial dynamics of the mutant populations. In the rest of this section, we describe results for only absorbing and reflective boundaries. We have run the same set of simulations for periodic boundaries and in all cases there is, as expected, no relationship between where a mutant arises and its survival and spread. Considering first the survival probability of mutants in relation to the edge, it is clear that when the boundary is absorbing, there is consistently lower survival at the edge (Table 1), whereas for reflective boundaries, the opposite is true. The difference in survival probabilities between the edge and elsewhere on the lattice is always greater for deleterious than for neutral mutants which, in turn, is greater than for beneficial mutants. For example, 5.96\% of deleterious mutants survive when they arise on an absorbing edge, compared with a mean across the entire range of $12.02 \%$, whereas corresponding values are 30.12 and $39.04 \%$ for neutral and 58.14 and $63.76 \%$ for beneficial mutants. When natal dispersal is more frequent, the relative differences between survival probabilities at the edge and the mean across space tend to become greater. This is most noticeable for deleterious mutants; for example, $18.15 / 11.44=1.59$ for $d=0.1$, $17.24 / 9.38=1.84$ for $d=0.2$ and $18.69 / 8.22=2.27$ for $d=0.3$. When we consider those mutants that have survived, we also observe edge effects influencing the maximum distance they travel, the number of patches they occupy and their total population size. For all fitness values, mutants have travelled further when they occur at the edge of a lattice with reflective boundaries, whereas they travel further when they occur away from the edge of a lattice with absorbing boundaries. Although deleterious mutants on average travel further than neutral or beneficial mutants, there is no clear relative difference between distances travelled by each fitness type depending on where they occur (Table 1, Figure 3). Surviving mutants that occur at the edge of an absorbing lattice always reach lower spatial extent and abundance than those occurring away from the edge. In general, this is similar on lattices with reflective boundaries; however, the effect is much reduced with this boundary type (Table 1, Figure 3 ).

The time between a patch being colonized and the mutant being introduced, $\Delta T$, has a major influence on the results regardless of where the mutant occurs relative to the edge or the boundary condition. In all cases, there is a rapid decrease in survival probability as $\Delta T$ increases. By the time $\Delta T=10$, the probability of a mutant surviving is very similar to the probability of a mutant surviving in a stationary population (Table 2 ). We show here only results for neutral and beneficial mutations, as simulations for deleterious mutations are too time-consuming due to the infrequency with which these mutants survive at higher $\Delta T$.

The leading edge of the range expansion is greatly affected by the boundary condition employed as well as the time since range expansion commenced (Figure 4). Absorbing boundary conditions result in the leading edge of the range expansion being convex in shape whereas reflective conditions lead to concave range 
Table 1 Survival probability, maximum displacement (Maxx), patch occupancy (Mpocc) and mutant population size (Mut size) for deleterious (Del.), neutral (Neu.) and beneficial (Ben.) mutations for three dispersal propensities

\begin{tabular}{|c|c|c|c|c|c|c|c|}
\hline \multirow[t]{3}{*}{ Fitness } & \multirow[t]{3}{*}{ Point of introduction } & \multicolumn{6}{|c|}{ Dispersal probability } \\
\hline & & \multicolumn{2}{|c|}{0.1} & \multicolumn{2}{|c|}{0.2} & \multicolumn{2}{|c|}{0.3} \\
\hline & & Abs. & Ref. & Abs. & Ref. & Abs. & Ref. \\
\hline \multicolumn{8}{|c|}{ Survival probability } \\
\hline \multirow[t]{3}{*}{ Del. } & 0 & 5.96 & 18.15 & 4.38 & 17.24 & 2.88 & 18.69 \\
\hline & 9 & 11.40 & 9.68 & 10.07 & 7.47 & 9.47 & 5.46 \\
\hline & Mean $\left(y_{0}-y_{9}\right)$ & 12.02 & 11.44 & 9.91 & 9.38 & 7.91 & 8.22 \\
\hline \multirow[t]{3}{*}{ Neu. } & 0 & 30.12 & 42.02 & 22.62 & 42.37 & 23.58 & 38.76 \\
\hline & 9 & 41.32 & 35.97 & 34.72 & 31.35 & 33.00 & 27.55 \\
\hline & Mean $\left(y_{0}-y_{9}\right)$ & 39.04 & 37.06 & 36.46 & 35.19 & 33.38 & 32.65 \\
\hline \multirow[t]{3}{*}{ Ben. } & 0 & 58.14 & 66.23 & 57.80 & 62.89 & 49.02 & 61.73 \\
\hline & 9 & 59.88 & 65.79 & 57.47 & 65.36 & 64.94 & 55.87 \\
\hline & Mean $\left(y_{0}-y_{9}\right)$ & 63.76 & 63.92 & 60.80 & 60.12 & 59.58 & 57.96 \\
\hline \multicolumn{8}{|l|}{$\operatorname{Maxx}$} \\
\hline \multirow[t]{3}{*}{ Del. } & 0 & 14.45 & 18.42 & 21.22 & 30.12 & 29.29 & 36.95 \\
\hline & 9 & 17.02 & 15.80 & 26.22 & 25.82 & 32.67 & 32.23 \\
\hline & Mean $\left(y_{0}-y_{9}\right)$ & 16.13 & 16.78 & 24.98 & 26.61 & 31.92 & 33.45 \\
\hline \multirow[t]{3}{*}{ Neu. } & 0 & 6.84 & 12.02 & 9.65 & 17.67 & 11.30 & 22.19 \\
\hline & 9 & 9.83 & 9.21 & 13.52 & 11.29 & 16.38 & 12.96 \\
\hline & Mean $\left(y_{0}-y_{9}\right)$ & 8.47 & 9.31 & 12.42 & 12.42 & 15.12 & 15.35 \\
\hline \multirow[t]{3}{*}{ Ben. } & 0 & 7.57 & 12.18 & 10.58 & 20.33 & 15.74 & 24.80 \\
\hline & 9 & 9.51 & 10.20 & 13.90 & 13.00 & 19.39 & 17.27 \\
\hline & Mean $\left(y_{0}-y_{9}\right)$ & 9.70 & 9.87 & 14.10 & 14.59 & 18.38 & 18.47 \\
\hline \multicolumn{8}{|l|}{ Мpocc } \\
\hline \multirow[t]{3}{*}{ Del. } & 0 & 43.17 & 60.99 & 59.39 & 120.58 & 104.88 & 182.36 \\
\hline & 9 & 84.49 & 68.73 & 150.04 & 112.78 & 182.62 & 145.08 \\
\hline & Mean $\left(y_{0}-y_{9}\right)$ & 65.77 & 63.48 & 114.96 & 112.81 & 156.60 & 147.46 \\
\hline \multirow[t]{3}{*}{ Neu. } & 0 & 32.28 & 64.65 & 54.75 & 125.33 & 67.09 & 180.93 \\
\hline & 9 & 73.55 & 63.63 & 129.43 & 93.84 & 166.45 & 114.14 \\
\hline & Mean $\left(y_{0}-y_{9}\right)$ & 55.44 & 60.00 & 97.02 & 94.15 & 133.21 & 131.52 \\
\hline \multirow[t]{3}{*}{ Ben. } & 0 & 67.92 & 114.55 & 148.75 & 271.29 & 262.36 & 404.72 \\
\hline & 9 & 146.59 & 147.33 & 293.46 & 288.03 & 477.15 & 447.41 \\
\hline & Mean $\left(y_{0}-y_{9}\right)$ & 125.38 & 126.77 & 254.84 & 261.28 & 391.81 & 399.00 \\
\hline \multicolumn{8}{|c|}{ Mut size } \\
\hline \multirow[t]{3}{*}{ Del. } & 0 & 290.09 & 425.68 & 340.34 & 786.39 & 599.27 & 1160.76 \\
\hline & 9 & 582.98 & 450.64 & 952.71 & 643.60 & 1137.72 & 769.38 \\
\hline & Mean $\left(y_{0}-y_{9}\right)$ & 449.57 & 422.69 & 734.29 & 690.41 & 959.68 & 846.72 \\
\hline \multirow[t]{3}{*}{ Neu. } & 0 & 195.86 & 444.89 & 275.11 & 771.92 & 271.05 & 1004.41 \\
\hline & 9 & 448.92 & 367.43 & 738.18 & 444.87 & 826.43 & 473.57 \\
\hline & Mean $\left(y_{0}-y_{9}\right)$ & 343.41 & 368.82 & 526.66 & 487.60 & 644.67 & 622.34 \\
\hline \multirow[t]{3}{*}{ Ben. } & 0 & 460.17 & 870.66 & 946.44 & 1998.02 & 1675.71 & 2850.65 \\
\hline & 9 & 1038.47 & 1034.10 & 1982.18 & 1941.26 & 3366.38 & 3079.84 \\
\hline & Mean $\left(y_{0}-y_{9}\right)$ & 901.78 & 908.53 & 1766.80 & 1815.63 & 2709.02 & 2752.69 \\
\hline
\end{tabular}

Data are shown for $y=0$ (edge), $y=9$ (core) and the mean across the range (mean $y_{0}-y_{9}$ ). Data are shown for absorbing (Abs.) and reflective (Ref.) boundaries.

fronts (Figure 4a). As anticipated, periodic boundary conditions do not lead to any change in the shape of the range front across the entire width (Figure 4a). For both absorbing and reflective boundary conditions, the shape of the range becomes more pronounced as the time since range expansion occurred increases, although the change in shape with time is greater for reflective boundary conditions (Figures $4 \mathrm{~b}$ and c). By 200 time steps after introduction, the shape of the expanding front has reached equilibrium (Figures $4 \mathrm{~b}$ and $\mathrm{c}$ ).

The percentage survival of mutations decreases with time for deleterious and neutral mutations (Figures 5a and $b$ ). As time since introduction increases, the percentage survival of deleterious mutations is minimal except in the case of mutations introduced at a reflective boundary (Figure 5a). The percentage survival of neutral mutations is dependent on where the novel mutant is introduced and the boundary condition employed (Figure $5 \mathrm{~b}$ ). The point of origin of the novel mutant and boundary condition leads to less variation in percentage survival for beneficial mutations (Figure 5c). For all three fitness types, mutants that survive for 200 time steps also have a high probability of surviving for up to 300 time steps (Figure 5). This effect is especially pronounced for deleterious mutants that first arise at a habitat edge with reflective boundary conditions.

\section{Influence of landscape features}

Edge effects on a heterogeneous landscape can result in substantial spatial variability in both the probability that a mutant will survive and the spatial dynamics of those that do survive. Figures 6 and 7 illustrate the results of simulations conducted on landscapes where a block of 
Table 2 The time between a patch being colonized and the mutant arising $(\Delta T)$ influences survival probabilities

\begin{tabular}{|c|c|c|c|c|c|c|c|}
\hline \multirow[t]{2}{*}{ Fitness } & \multirow{2}{*}{$\begin{array}{l}\text { Point of } \\
\text { introduction }\end{array}$} & \multicolumn{6}{|c|}{$\Delta T$} \\
\hline & & 0 & 1 & 3 & 6 & 10 & ST \\
\hline \multicolumn{8}{|c|}{ Absorbing } \\
\hline \multirow[t]{3}{*}{ Neu. } & 0 & 30.12 & 20.45 & 10.26 & 4.98 & 3.46 & 3.14 \\
\hline & 9 & 39.06 & 27.86 & 12.44 & 5.23 & 3.08 & 2.32 \\
\hline & Mean $\left(y_{0}-y_{9}\right)$ & 37.80 & 27.17 & 12.87 & 5.53 & 3.15 & 2.58 \\
\hline \multirow[t]{3}{*}{ Ben. } & 0 & 58.14 & 46.95 & 38.76 & 21.55 & 18.32 & 20.83 \\
\hline & 9 & 67.11 & 58.14 & 37.31 & 24.94 & 20.96 & 21.55 \\
\hline & Mean $\left(y_{0}-y_{9}\right)$ & 64.69 & 55.34 & 37.72 & 25.10 & 20.33 & 20.01 \\
\hline \multicolumn{8}{|c|}{ Reflective } \\
\hline \multirow{3}{*}{ Neu. } & 0 & 42.02 & 30.58 & 15.75 & 7.10 & 3.17 & 2.40 \\
\hline & 9 & 42.19 & 27.93 & 14.37 & 5.16 & 2.89 & 2.81 \\
\hline & Mean $\left(y_{0}-y_{9}\right)$ & 39.04 & 27.55 & 13.59 & 59.11 & 34.28 & 2.71 \\
\hline \multirow[t]{3}{*}{ Ben. } & 0 & 66.23 & 52.91 & 38.17 & 25.64 & 22.22 & 21.74 \\
\hline & 9 & 66.67 & 52.08 & 36.76 & 21.10 & 23.47 & 18.83 \\
\hline & Mean $\left(y_{0}-y_{9}\right)$ & 63.25 & 54.47 & 37.57 & 24.87 & 21.12 & 19.83 \\
\hline
\end{tabular}

The column labelled ST shows equivalent results for a stationary population. Data are shown for $y=0$ (edge), $y=9$ (core) and the mean across the range (mean $\left.y_{0}-y_{9}\right)$ for neutral (Neu.) and beneficial (Ben.) mutations.

habitat has been made unsuitable within an otherwise intact region and a narrow corridor of habitat connects two larger areas of suitable habitat, respectively. In both these figures, the greyscale of each cell shows the outcome for mutants that have been introduced to that cell. The location at which a deleterious mutation arises has a marked effect on the genotype's survival probability; there is a clear pattern where the probability of survival is substantially reduced both behind and in front of the region of unsuitable habitat. Although the observed pattern for neutral mutations is less significant, those that are introduced directly behind, and in front of, the area of unsuitable habitat have lower survival probability. There is very little pattern in the spatial variation of survival probabilities for beneficial mutations. The point of introduction of those deleterious and neutral mutations that survive has a significant effect on population size and displacement. When individuals are introduced into the regions on either side of the unsuitable habitat, there is a substantial reduction in the size of any resulting populations and their migration distance. Although no pattern in survival probability is observed for beneficial mutations, there is a considerable decrease in the resulting mutant population size when mutations are introduced on either side of the unsuitable habitat area. There is also a noteworthy reduction in the displacement of individuals when the beneficial mutation is initiated behind the area of unsuitable habitat. An edge effect is observed, for each of the mutation types, which is dependent on the boundary condition employed. For deleterious and neutral mutations, absorbing boundaries lead to a decrease in survival probability at the edge of the lattice. The use of reflective boundaries has the opposite effect although this pattern is reduced for neutral mutations. No obvious effects of boundary condition on the survival probability of beneficial mutations are observed. Absorbing boundaries give rise to a substantial decrease in the population size of surviving mutations at the edge of the lattice for each mutation type. A slight increase in population size is
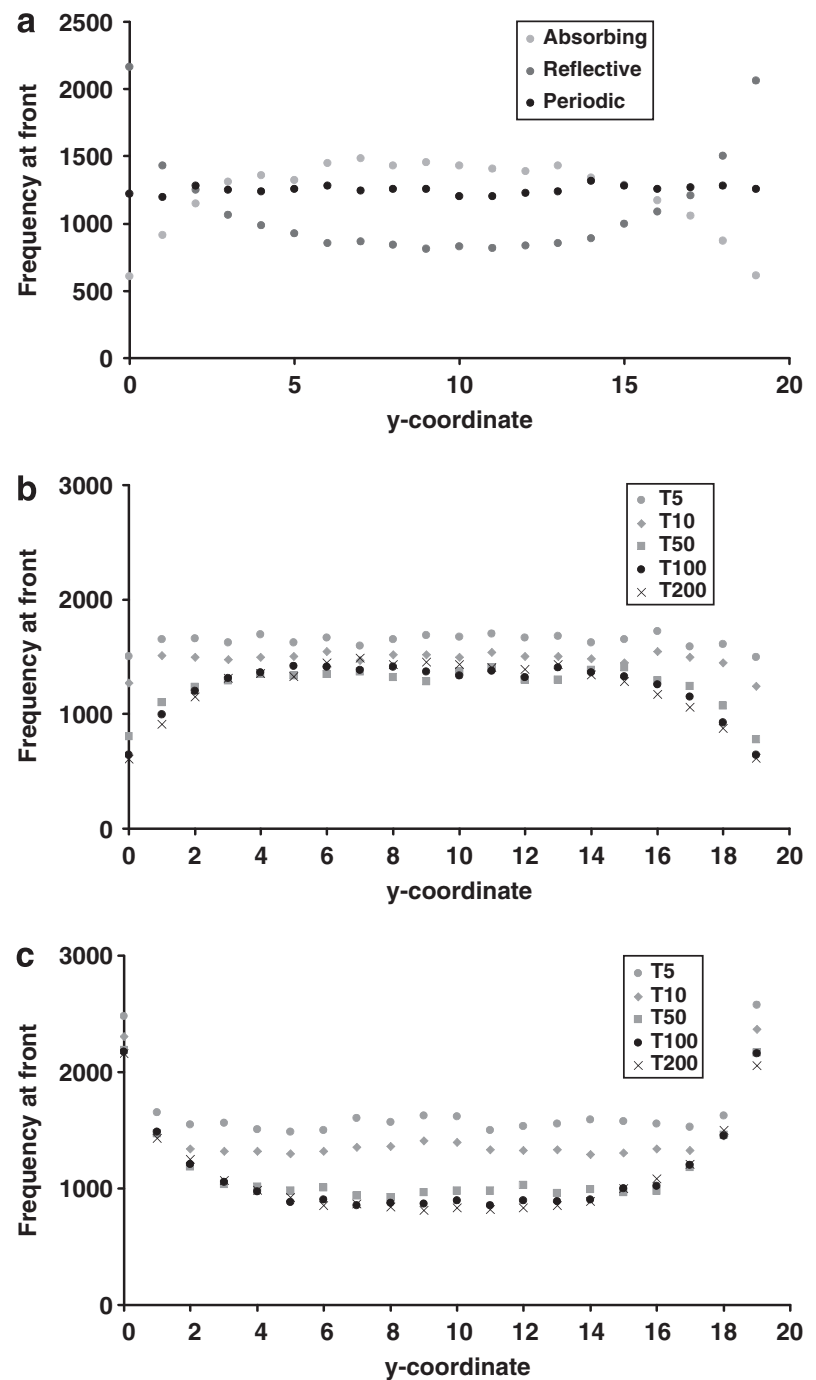

Figure 4 The shape of the expanding range front is affected by boundary condition and time since population established. (a) The frequency that each $y$-coordinate was at the front of the expanding range 200 generations after expansion has occurred for all three boundary conditions. (b and $\mathbf{c}$ ) The frequency that each $y$ coordinate was at the front of range after different time steps for absorbing and reflective boundaries, respectively. Points show the frequency (out of 10000 repeat simulations) that each $y$-coordinate was at the front of the expanding range 100 generations after expansion started. These results were generated for $K=10$ and $R=1.8$

observed when reflective boundary conditions are applied. The displacement of individuals at the edge of the lattice is affected to a lesser extent by boundary condition. Here, a slight decrease and increase in the centroid $x$-coordinate is observed at the periphery for absorbing and reflective boundaries, respectively (Figure 6).

Simulations conducted using a habitat corridor also lead to significant spatial variation in survival probability and mutation dynamics with the greatest effects observed for deleterious mutations. Deleterious mutations are far more likely to survive when they arise in the vicinity of the corridor, and especially when their origin is located just beyond the corridor. This pattern is less pronounced for neutral mutations and not evident for 

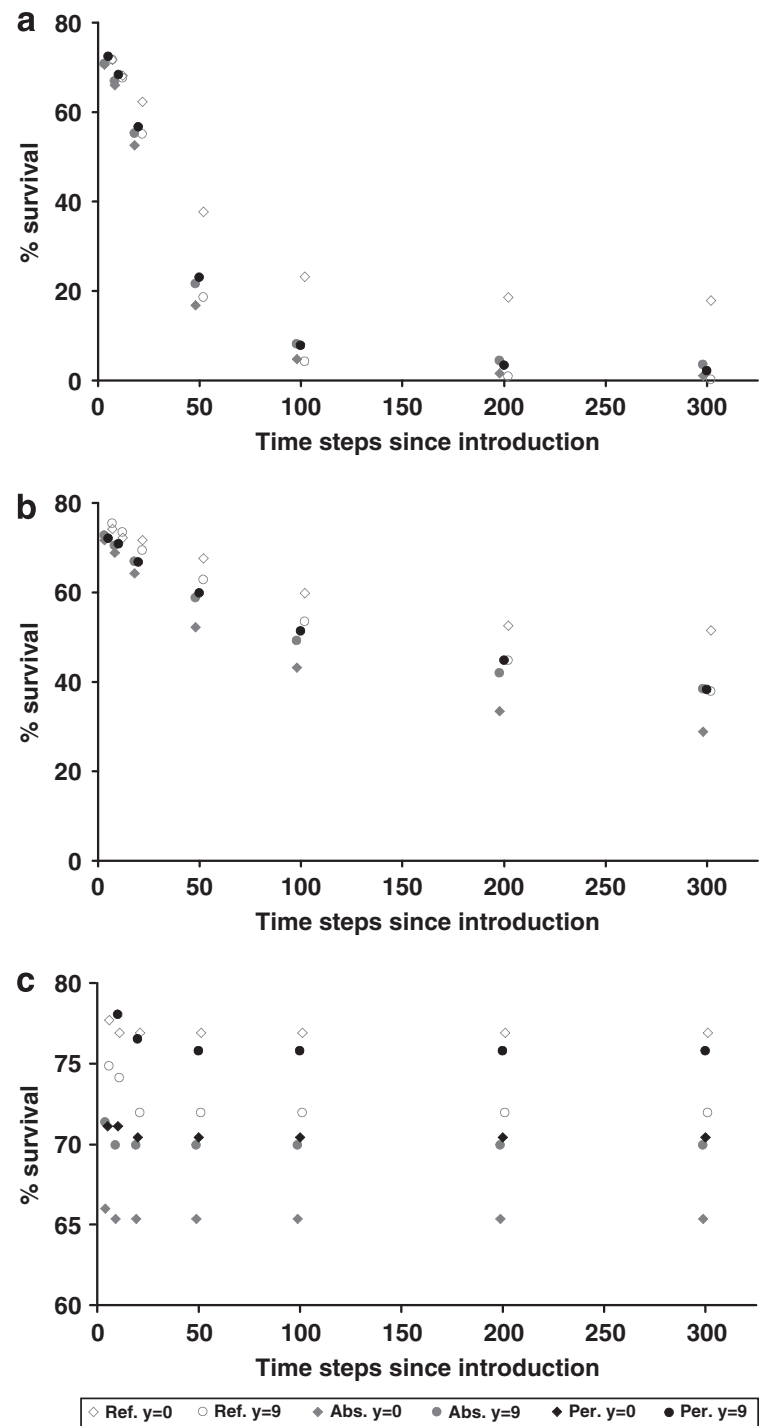

Figure 5 The amount of time since introduction of the novel mutant and boundary condition both affect the survival of mutations. Here, we report the percentage survival of mutations for absorbing (Abs.), reflective (Ref.) and periodic (Per.) boundary conditions after different time steps since introduction of the novel mutant. Here, the data points are offset slightly along the $x$ axis to improve readability. $(\mathbf{a}-\mathbf{c})$ The survival of introduced deleterious, neutral and beneficial mutations, respectively. In all three graphs, the percentage survival of mutations that are introduced at $y=0$ (at the boundary) and $y=9$ (in the centre of the range) are shown for absorbing and reflective boundaries. In $\mathbf{a}$ and $\mathbf{b}$, the only results at $y=9$ for periodic boundary conditions is shown as there was very little difference to those at $y=0$. The results for both $y=0$ and $y=9$ under periodic boundary conditions are shown in c. Again $K=10$ and $R=1.8$.

beneficial mutations (Figure 7). The population size and maximum displacement of surviving mutants is influenced by their origin for all fitness types, with greatest population abundances and displacements always found for those mutants that arise near the corridor. Edge effects due to differences between the boundary conditions are less apparent for this landscape structure. For each map, the maximum and minimum values across all patches on the landscapes are shown in the accompanying tables.

\section{Discussion}

The results presented in this article clearly demonstrate the importance of edge effects, boundary conditions and landscape structure in determining the fate of mutations that occur during a period of population range expansion. Our results are consistent with previous studies in demonstrating that mutation surfing can play an important role in determining the survival probability and spatial distribution of mutants occurring at a front (Edmonds et al., 2004; Klopfstein et al., 2006; Travis et al., 2007). These new results serve to highlight that both the survival probability and the characteristics of surviving mutants are highly dependent on landscape structure and the dispersal behaviour of individuals around habitat edges.

Habitat boundaries introduce considerable spatial variation in the survival probabilities of mutations depending on where they occur. With absorbing boundaries, mutations that arise at, or near, an edge are less likely to survive. This is due to two effects. First, individuals at the edge suffer from the risk of dispersing over the edge and dying, and this elevated risk of mortality increases the probability that a mutation becomes extinct almost immediately. Second, with absorbing boundary conditions, the leading edge of the range expansion is most often away from the edge towards the centre of the lattice (Figure $4 a$ ), which is due to additional mortality at absorbing edges leading to less rapid population growth. Mutations are most likely to survive when they occur at, or very close to, the leading edge, as it is only then that they and their descendants are most likely to remain at the front and benefit from reduced competition (Klopfstein et al., 2006; Travis et al., 2007; Burton and Travis, 2008). The first of these effects applies equally to mutations regardless of their fitness effect, but the second has a much greater influence on deleterious mutations, as their survival is much more dependent on them surfing the expanding wave front than it is for neutral or beneficial mutations. This pattern is reversed for reflective boundaries and there is a higher survival probability of mutations that occur at the edge. Reflective boundaries do not impose an additional mortality cost, but instead promote dispersal parallel to the edge (Figure 4a). Here individuals that would otherwise disperse off the edge move at random to a patch adjacent to the natal deme. Consider an individual with a dispersal probability of 0.1 in an edge cell right at the expanding front. With absorbing boundary conditions, its probability of colonizing an empty patch along the habitat edge is 0.0250 . However, with reflective boundary conditions, this is increased to 0.0375 . This increases the rate of range expansion along an edge and makes it more likely that mutations arising there will surf, thus increasing their survival probability. Again, because surfing is most important in determining the survival of deleterious mutations, this leads to a greater effect on their survival probability and population dynamics than it does for neutral and beneficial mutations.

When the characteristics of surviving mutations are considered, edge effects are found to influence the abundance, spatial extent and spread of all three fitness types investigated. Surviving mutations with an origin at, or near, the edge of lattices with absorbing boundaries 
Del. Abs.
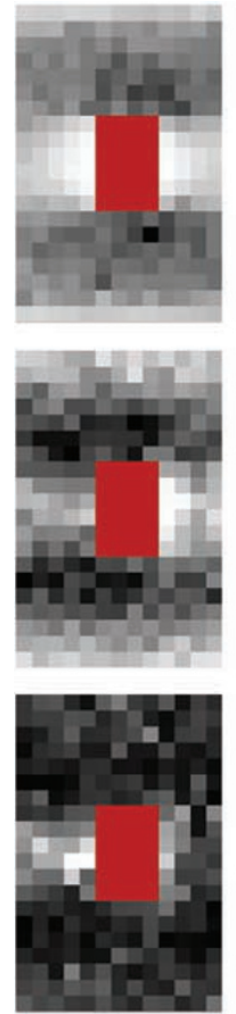

Del. Ref.
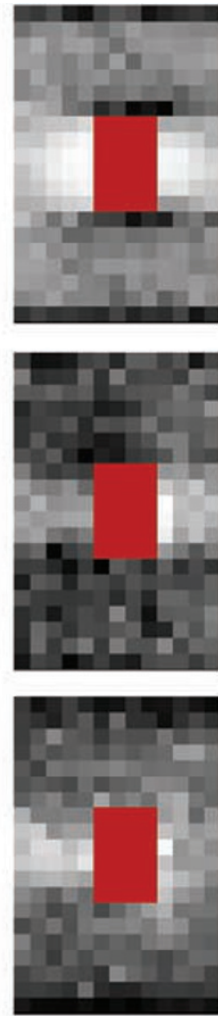

Neu. Abs.
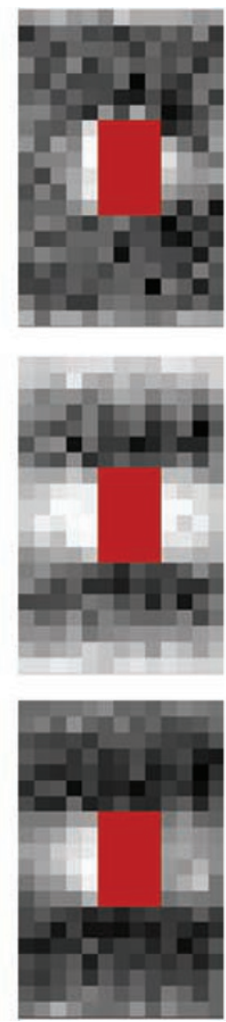

Neu. Ref.
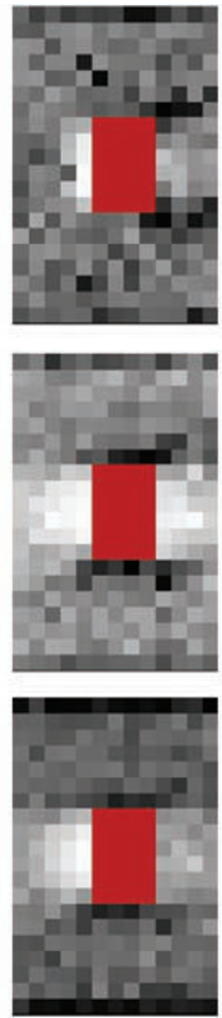

Ben. Abs.
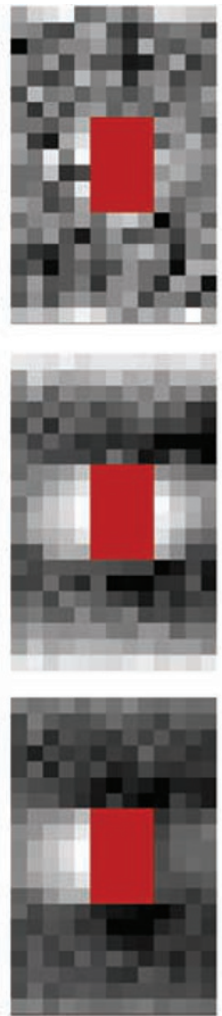

Ben. Ref.
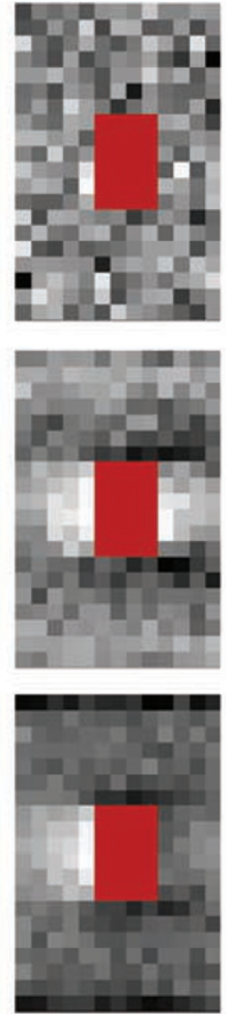

Max survival probability
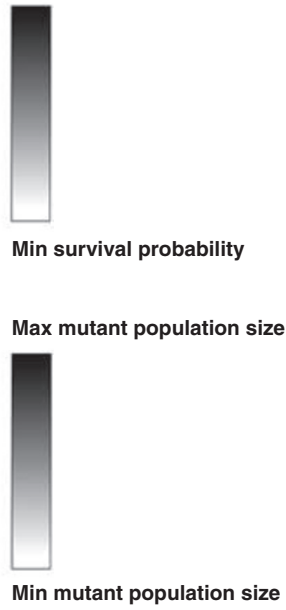

Max centroid x-coordinate

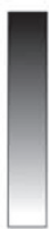

Min centroid x-coordinate

\begin{tabular}{|c|c|c|c|c|c|c|c|c|c|c|c|c|}
\hline \multirow[b]{3}{*}{ Statistic } & \multicolumn{12}{|c|}{ Finess of mutant and boundary condition } \\
\hline & Del. Abs. & & Del. Ref & & Neu. Abs & & Neu. Ref & & Ben. Abs. & & Ben. Ref. & \\
\hline & $\overline{\operatorname{Max}}$ & Min & Max & Min & Max & Min & Max & Min & $\operatorname{Max}$ & Min & Max & Min \\
\hline Survival probability & 0.276 & 0.008 & 0.244 & 0.017 & 0.508 & 0.182 & 0.508 & 0.211 & 0.735 & 0.521 & 0.758 & 0.532 \\
\hline Mutant population size & 756.170 & 186.270 & 632.590 & 152.690 & 723.320 & 64.270 & 703.230 & 85.530 & 1218.500 & 370.920 & 1239.960 & 485.590 \\
\hline Centroid $x$-coordinate & 12.113 & 6.167 & 13.851 & 7.701 & 6.008 & -0.851 & 6.786 & -0.381 & 3.494 & -1.633 & 4.098 & -1.362 \\
\hline
\end{tabular}

Figure 6 Introducing simple landscape structure leads to considerable spatial variability in survival probability, mutant population size and spatial spread (centroid $x$-coordinate). The simulations were conducted on lattices of the same dimensions as elsewhere, but only the portion of the lattice where mutations were introduced $(100 \leqslant x \leqslant 112$ and $0 \leqslant y \leqslant 19)$ is shown-representing the region around unavailable habitat. The greyscale of each cell shows how likely a mutant, arising at that location, is to survive, increase in abundance and spread. For each location, the model is run until 100 mutants survive for 100 time steps. The red region represents unavailable habitat. Results from simulations with deleterious (Del.), neutral (Neu.) and beneficial (Ben.) mutants for each of absorbing (Abs.) and reflective (Ref.) boundary conditions are shown. The maximum and minimum values are shown in the table for each of the three statistics illustrated in this set of maps. Again $K=10$ and $R=1.8$.

typically reach lower population size, and do not travel as far. This is for the same reasons that mutations arising at these edges have depressed survival probabilities. The absorbing boundaries impose an additional mortality cost, and mutations occurring there are less likely to be on the extreme edge of the front. Additionally, because mutations arising near an edge can only spread along and away from that edge, they will on average reach lower abundance than those arising in the centre of the lattice that can spread in all directions. Reflective boundary conditions lead to greater $x$-displacements of those mutations that arise close to an edge. This is true for all fitness types, as reflective edges promote increased dispersal in the direction of range expansion. This effect also leads to surviving deleterious and neutral mutants obtaining higher population abundance when they arise close to the edge. However, the population size of surviving beneficial mutants is affected much less by where they occur in relation to a reflecting edge, and this is because their survival and subsequent dynamics are much less dependent on surfing behaviour. Except for mutations with negative fitness effects, most surviving mutations do not surf (Klopfstein et al., 2006; Travis et al., 2007) and non-surfing beneficial mutants will obtain higher abundances when they arise towards the centre of the lattice and can spread, unconstrained by edges, in all directions. Beneficial mutants will typically spread in all directions from their origin but, in many cases, their rate of expansion through populations of the wild type is slower than the range expansion, and hence they do not surf.

In contrast to most recently published work, we have identified substantial effects of different boundary conditions. Results from previous simulations, investigating spatial population dynamics, typically report little or no overall effect in results obtained with alternative boundary conditions (Molofsky et al., 2002; Leblois et al., 2006). Other works have considered differences in results a little further. Kean and Barlow (2000) found '...little effect on metapopulation dynamics, except that absorbing boundaries imposed additional mortality on the metapopulation...'. Etienne et al. (2002) also reported 

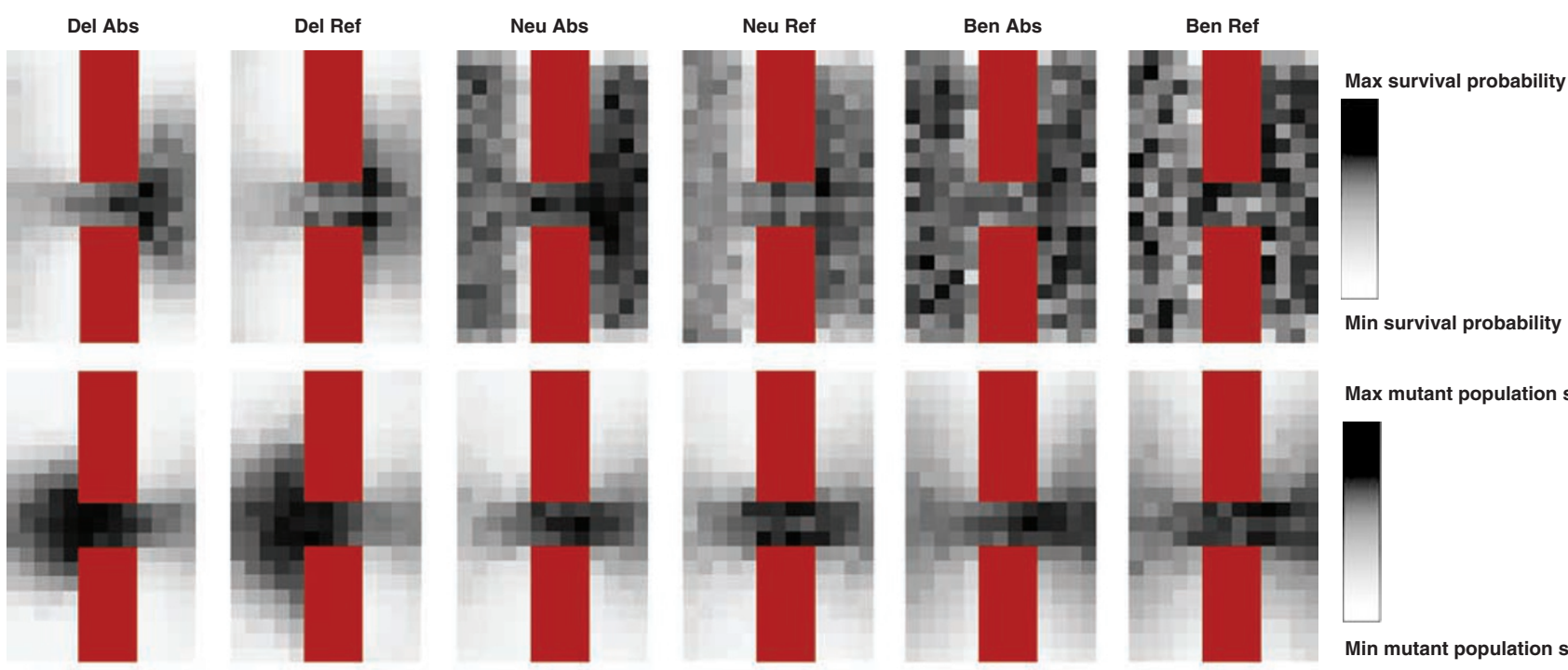

Max mutant population size
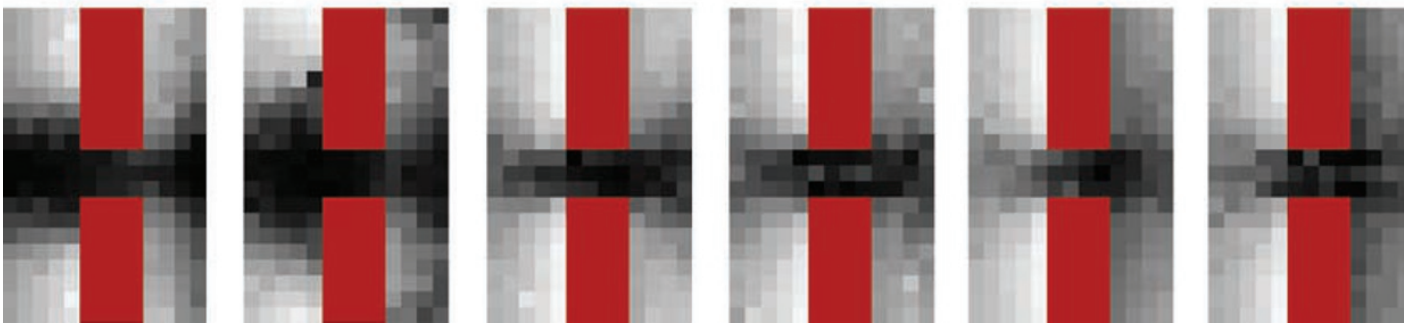

Min mutant population size

\begin{tabular}{|c|c|c|c|c|c|c|c|c|c|c|c|c|}
\hline \multirow[b]{3}{*}{ Statistic } & \multicolumn{12}{|c|}{ Finess of mutant and boundary condition } \\
\hline & \multicolumn{2}{|c|}{ Del. Abs. } & \multicolumn{2}{|c|}{ Del. Ref. } & \multicolumn{2}{|c|}{ Neu. Abs. } & \multicolumn{2}{|c|}{ Jeu. Ref. } & \multicolumn{2}{|c|}{ Ben. Abs. } & \multicolumn{2}{|c|}{ Ben. Ref. } \\
\hline & Max & Min & Max & Min & Max & Min & Max & Min & Max & Min & Max & Min \\
\hline Survival probability & 0.4484 & 0.0016 & 0.4274 & 0.0022 & 0.5405 & 0.1473 & 0.6452 & 0.1572 & 0.7634 & 0.4405 & 0.7353 & 0.5128 \\
\hline Mutant population size & 2028.37 & 18.58 & 2086.83 & 7.66 & 221.51 & 7.13 & 198.51 & 9.14 & 234.85 & 28.48 & 227.87 & 36.5 \\
\hline Centroid $\mathrm{x}$-coordinate & 19.71 & 0.26 & 11.48957 & -1.9444 & 7.2587 & -1.268 & 7.3454 & -1.4508 & 5.749 & -2.0883 & 5.2717 & -2.593 \\
\hline
\end{tabular}

Figure 7 A habitat corridor introduces strong spatial variation in the fate of mutants. Other than the shape of the habitat feature, all simulation details are the same as in Figure 6.

slight differences in their results under varying boundary conditions, demonstrating that reflective and periodic boundaries lead to some results that were quantitatively different but qualitatively similar. Also, changes in population dynamics were observed for simulations using absorbing boundaries. Our findings emphasize that under range expansion conditions, there is an increased importance of considering the effect of boundary conditions, as they can significantly influence population dynamics and therefore the outcome of simulations.

The use of corridors connecting distinct and spatially separated habitat patches has become a much-used strategy in conservation biology (for example, Hudgens and Haddad, 2003). Corridors offer the potential benefit of increasing movement rates of individuals and genes between patches and can thus protect the demographic and genetic integrity of spatially extended populations existing on fragmented landscapes (Brier and Noss, 1998). Corridors can also have potential negative impacts such as increasing the spread of diseases or invasive predators or pests (Hess, 1994). Few theoretical works have addressed the potential influence of corridors on genetic processes. One recent simulation study illustrated how a habitat corridor linking two patches (one permanently suitable and one ephemeral) can have substantial effects on the fixation probabilities of novel alleles (Orrock, 2005). During periods of climate change, habitat corridors will assume another important conservation role: facilitating the spread of species into regions as they become suitable, thus increasing the rate of range shifting (Halpin, 1997; Collingham and Huntley, 2000). Although corridors are likely to increase migration rates of dispersive organisms, questions remain as to their likely effectiveness for sessile or sedentary organisms (Collingham and Huntley, 2000). Orrock's work (2005) shows that corridors can have important genetic effects in the absence of climate change. As yet, we have little understanding of their likely impact on genetics during periods of range shifting. Our results indicate that corridors can introduce substantial spatial variation in the fate of mutations depending on where they arise during a range expansion. In particular, when deleterious mutations occur just in front of, along or (especially) just beyond a habitat corridor, they have a much greater probability of surviving. Those that survive are likely to surf to substantial abundance and spatial extent. As future work considers questions regarding the use of corridors as a conservation strategy geared towards facilitating species migrations, there needs be a consideration not only of demographic, but also genetic processes. Important questions are how a network of 
habitat patches connected by corridors may impact on the genetic load of a population as it spreads across the network, and how the risk of potentially harmful increases in load can be minimized by careful consideration of network structure-for example, corridor widths.

An increasing number of simulation studies are investigating the dynamics and spatial spread of neutral mutations that occur during a period of range expansion (Ibrahim et al., 1996; Austerlitz and Garnier-Géré, 2003; Edmonds et al., 2004; Bialozyt et al., 2006; Klopfstein et al., 2006) but, to date, far less works have considered the fate of non-neutral mutations. It has recently been demonstrated that the surfing phenomenon observed by Edmonds et al. (2004) and Klopfstein et al. (2006) is likely to lead to the substantially increased survival and spread of deleterious mutants at the front (Travis et al., 2007; Burton and Travis, 2008), and the results presented here corroborate with those results. Whereas previous works have focussed on using a centroid statistic to describe spread, here we have used spatial extent (as measured by the number of patches occupied) and total population size, in addition to looking at the maximum $x$-displacement obtained by a surviving mutation. These statistics serve to illustrate the dynamics more clearly and highlight that surviving deleterious mutants may, on average, occupy more patches and have higher total abundance than an average surviving neutral mutant (Table 1, Figure 3). As a greater range of methods are developed to describe empirical patterns of quantitative genetic variation (for example, Mauricio, 2001; Erickson et al., 2004), there will be considerable potential to use these, together with data on neutral markers, to improve inferences regarding past range expansions. These empirical data can only give us a picture of mutations that have survived until today, and simulations that can produce expected patterns of surviving neutral and non-neutral genetic diversity will be needed if we are to best use the additional information that new quantitative genetic methods will provide. Looking simultaneously at clines in neutral and non-neutral genetic diversity should markedly improve our capacity to infer the patterns of past range expansions.

To improve both our capacity for inference of past range expansion and our ability to predict the genetic consequences of current range shifts, we need to begin relaxing some of the simplifying assumptions that are very often made in simulation studies of the type presented here. We have assumed strict nearest-neighbour dispersal, yet we already know from other studies (for example, Ibrahim et al., 1996; Bialozyt et al., 2006) that the shape of a dispersal kernel can have a major influence on the patterns of neutral genetic diversity that emerge during range expansions. To date, studies describing the mutation surfing phenomenon have assumed nearest-neighbour dispersal (Edmonds et al., 2004; Klopfstein et al., 2006; Travis et al., 2007; Burton and Travis, 2008) and future work should consider how differently shaped dispersal kernels might influence the probability of mutants surfing, depending both on when they occur relative to the front and on their fitness effect.

Existing studies on mutation surfing have assumed very simple within-patch dynamics and, although it is likely that the surfing dynamics will be qualitatively similar if other functions of local dynamics are used, a study considering the potential for quantitative differ- ences resulting from the choice of within-population dynamics would be valuable. In particular, recent work has shown how over-compensating density dependence can have a major influence on the spatial population dynamics of range expansions (Best et al., 2007) and it would be interesting to establish how it might also impact the surfing behaviour of novel mutants. Similarly, density-dependent dispersal may have a substantial impact on surfing dynamics. Intuitively, we would anticipate that it would reduce the likelihood of deleterious mutants surfing for long periods, but this represents a further area worthy of thorough investigation. We have considered the dynamics of mutations in a spatially structured environment, but we do not consider true metapopulation dynamics, as local extinction events are rare. Mathematical approaches have been developed in the population genetics literature, with the purpose of exploring the dynamics of mutations within metapopulations (for example, Whitlock and Barton, 1997; Pannell and Charlesworth, 2000), and it would be interesting to extend these to explore range expansions.

We have assumed that mutants have different relative juvenile competitive abilities. A consequence of this is that deleterious and beneficial mutations are effectively neutral within a deme up to the point where $K$ is reached, due to the lack of competition. However, fitness could equally influence other life history characteristics, and we suggest that future work considering the dynamics of novel mutations that impact reproductive rate and dispersal propensity would be interesting extensions. This would lead to a greater understanding of the evolution of life history during range expansions, a topic of considerable current interest (for example, Thomas et al., 2001; Simmons and Thomas, 2004; Phillips et al., 2006). Most studies have considered a haploid genetic system and, although this represents a sensible assumption for neutral variability with maternal inheritance such as mitochondrial DNA, it is inappropriate if we are interested in adaptive genetic variability in diploid organisms. If we should be able to answer important conservation-relevant questions regarding the evolutionary potential of range shifting versus stationary populations, we will need to model diploid organisms and begin to incorporate multiple loci and genetic details such as epistasis, pleiotropy and hitchhiking (Orrock, 2005). There is also an opportunity to link emerging ideas in this area of population genetics with well-established methodologies in landscape ecology. This would enable simulations of real species on real landscapes where the ecology of the species, including habitat requirements and preferences, are described in some detail and the characteristics of the landscape are incorporated through the use of Geographic Information Systems (GIS) (for example, Hammersh $\varnothing$ j et al., 2006). This would facilitate a shift from purely strategic models aimed at providing generic insights to rather more tactical models that might, for example, inform the design of a reserve network with the aim of protecting the genetic integrity of a particular group of species as they shift their ranges in response to climate change.

\section{References}

Austerlitz F, Garnier-Géré PH (2003). Modelling the impact of colonisation on genetic diversity and differentiation of forest 
trees: interaction of life cycle, pollen flow and seed long distance dispersal. Heredity 90: 282-290.

Bell JR, Bohan DA, Shaw EM, Weyman GS (2005). Ballooning dispersal using silk: world fauna, phylogenies, genetics and models. Bull Entomol Res 95: 69-114.

Best AS, Johst K, Münkemüller T, Travis JMJ (2007). Which species will successfully track climate change? The influence of intraspecific competition and density dependent dispersal on range shifting dynamics. Oikos 116: 1531-1539.

Bialozyt R, Ziegenhagen B, Petit RJ (2006). Contrasting effects of long distance seed dispersal on genetic diversity during range expansion. J Evol Biol 19: 12-20.

Bonte D, Lens L, Maelfait JP, Hoffmann M, Kuijken E (2003). Patch quality and connectivity influence spatial dynamics in a dune wolfspider. Oecologia 135: 227-233.

Brier P, Noss RF (1998). Do habitat corridors really provide connectivity? Cons Biol 12: 1241-1252.

Burton OJ, Travis JMJ (2008). The frequency of fitness peak shifts is increased at expanding range margins due to mutation surfing. Genetics 179: 941-950.

Cadenasso ML, Pickett STA, Weathers KC, Bell SS, Benning TL, Carreiro MM et al. (2003). An interdisciplinary and synthetic approach to ecological boundaries. BioScience 53: 717-722.

Chapman DS, Dytham C, Oxford GS (2007). Landscape and fine-scale movements of a leaf beetle: the importance of boundary behaviour. Oecologia 154: 55-64.

Collingham YC, Huntley B (2000). Impacts of habitat fragmentation and patch size upon migration rates. Ecol Appl 10: 131-144.

Comins HN, Hassell MP, May RM (1992). The spatial dynamics of host-parasitoid systems. J Anim Ecol 61: 735-748.

Edmonds CA, Lillie AS, Cavalli-Sforza LL (2004). Mutations arising in the wave front of an expanding population. Proc Natl Acad Sci USA 101: 975-979.

Erickson DL, Fenster CB, Sten $\varnothing$ len HK, Price D (2004) Quantitative trait locus analyses and the study of evolutionary processes. Mol Ecol 13: 2505-2522.

Etienne R, Wertheim B, Hemerik L, Schneider P, Powell J (2002). The interaction between dispersal, the Allee effect and scramble competition affects population dynamics. Ecol Model 148: 153-168.

Fagan WF, Canrell RS, Cosner C (1999). How habitat edges change species interactions. Am Nat 153: 165-182.

Halpin PN (1997). Global climate change and natural-area protection: management responses and research directions. Ecol Appl 7: 828-843.

Hammersh $ø$ j M, Travis JMJ, Stephenson CM (2006). Incorporating evolutionary processes into a spatially-explicit model: exploring the consequences of mink-farm closures in Denmark. Ecography 29: 465-476.

Hänfling B, Kollmann J (2002). An evolutionary perspective of biological invasions. Trends Ecol Ecol 17: 22-27.

Hess GR (1994). Conservation corridors and contiguous disease: a cautionary note. Cons Biol 8: 256-262.

Hudgens BR, Haddad NM (2003). Predicting which species will benefit from corridors in fragmented landscapes from population growth models. Am Nat 161: 808-820.

Ibrahim KM, Nichols RA, Hewitt GM (1996). Spatial patterns of genetic variation generated by different forms of dispersal during range expansion. Heredity 77: 282-291.

Kean JM, Barlow ND (2000). The effects of density-dependence and local dispersal in individual-based stochastic metapopulations. OIKOS 88: 282-290.
Klopfstein S, Currat M, Excoffier L (2006). The fate of mutations surfing on the wave of a range expansion. Mol Biol Evol 23: 482-490.

Knopp T, Kano JM, Crochet P-A, Merilä J (2007). Contrasting levels of variation in neutral and quantitative genetic loci on island populations of moor frogs (Rana arvalis). Cons Genet 8 : $45-56$.

Lambrinos JG (2004). How interactions between ecology and evolution influence contemporary invasion dynamics. Ecology 85: 2061-2070.

Leblois R, Estoup A, Streiff R (2006). Genetics of recent habitat contraction and reduction in population size: does isolation by distance matter? Mol Ecol 15: 3601-3615.

Lee CE (2002). Evolutionary genetics of invasive species. Trends Ecol Evol 17: 386-391.

Manel S, Schwartz MK, Luikart G, Taberlet P (2003). Landscape genetics: combining landscape ecology and population genetics. Trends Ecol Evol 18: 189-197.

Mauricio R (2001). Mapping quantitative trait loci in plants: uses and caveats for evolutionary biology. Nature Rev Genet 2: 370-381.

McInerny G, Travis JMJ, Dytham C (2007). Range shifting on a fragmented landscape. Ecol Inf 2: 1-8.

Molofsky J, Bever JD, Antonovics J, Newman T (2002). Negative frequency dependence and the importance of spatial scale. Ecology 83: 21-27.

Orrock JL (2005). Conservation corridors affect the fixation of novel alleles. Cons Gen 6: 623-630.

Pannell JR, Charlesworth B (2000). Effects of metapopulation processes on measures of genetic diversity. Phil Trans $R$ Soc Lond B 355: 1851-1864.

Parmesan C, Yohe G (2003). A globally coherent fingerprint of climate change impacts across natural systems. Nature 421: 37-42.

Phillips BL, Brown GP, Webb JK, Shine R (2006). Invasion and the evolution of speed in toads. Nature 439: 803.

Simmons AD, Thomas CD (2004). Changes in dispersal during species' range expansions. Am Nat 164: 378-395.

Stevens VM, Leboulenge E, Wesselingh RA, Baguette M (2006). Quantifying functional connectivity: experimental assessment of boundary permeability for the natterjack toad (Bufo calamita). Oecologia 150: 161-171.

Storfer A, Murphy MA, Evans JS, Goldberg CS, Robinson S, Spear SF et al. (2007). Putting the 'landscape' in landscape genetics. Heredity 98: 128-142.

Tackenberg O, Poschlod P, Bonn S (2003). Assessment of wind dispersal potential in plant species. Ecol Mon 73: 191-205.

Thomas CD, Bodsworth EJ, Wilson RJ, Simmons AD, Davies ZG, Musche M et al. (2001). Ecological and evolutionary processes at expanding range margins. Nature 411: 577-581.

Travis JMJ, Dytham C (2002). Dispersal evolution during invasions. Evol Ecol Res 4: 1119-1129.

Travis JMJ, Münkemüller T, Burton OJ, Best A, Dytham C, Johst K (2007). Deleterious mutations can surf to high densities on the wave front of an expanding population. Mol Biol Evol 24: 2334-2343.

Wallenius KT (1963). Biased Sampling: The Non-Central Hypergeometric Probability Distribution. Ph.D. thesis, Stanford University.

Whitlock MC, Barton NH (1997). The effective size of a subdivided population. Genetics 146: 427-441.

With KA (2002). The landscape ecology of invasive spread. Cons Biol 16: 1192-1203. 